\title{
Alterations in Co-abundant Bacteriome in Colorectal Cancer and Persistent Microbial Dysbiosis After Surgery
}

\author{
Chin-Wen Png \\ National University of Singapore \\ Yong-Kang Chua \\ National University Hospital \\ Jia-Hao Law \\ National University Hospital \\ Yongliang Zhang \\ National University of Singapore \\ Ker-Kan Tan ( $\nabla$ surtkk@nus.edu.sg ) \\ National University Hospital
}

\section{Research Article}

Keywords: colorectal cancer, microbiome, gut microbiome, colorectal cancer screening, colorectal cancer surgery

Posted Date: December 16th, 2021

DOI: https://doi.org/10.21203/rs.3.rs-1130837/v1

License: (c) (i) This work is licensed under a Creative Commons Attribution 4.0 International License. Read Full License 


\section{Abstract}

Objective

There is growing interest in the role of gut microbiome in colorectal cancer (CRC), ranging from screening to disease recurrence. Our study aims to identify microbial markers characteristic of CRC and to examine if changes in bacteriome persist after surgery.

Design

49 fecal samples from non-cancer (NC) individuals and CRC patients, before and after surgery, were collected for analysis by bacterial 16S rRNA gene sequencing.

Results

Bacterial richness and diversity were reduced in the CRC patients compared to NC individuals. Pro-carcinogenic bacteria such as Bacteroides fragilis and Odoribacter splanchnicus were increased in pre-op CRC compared to NC group. These differences were no longer observed after surgery. Comparison between post- and pre-op CRC showed increased abundance of probiotic bacteria after surgery. Concomitantly, bacteria associated with CRC progression were observed to have increased after surgery, implying persistent dysbiosis.

\section{Conclusion}

Microbiome signatures characteristic of CRC likely comprise a set of significant alteration in specific bacterial strains. Elements of these dysbiotic signatures persists even after surgery, suggesting possible field-change in remnant nondiseased colon. Future studies should seek to examine if these signatures are also associated with pre-malignant colonic polyps and, explore if these profiles can be used to guide CRC screening and surveillance.

\section{Introduction}

The human microbiome is a second genome of the human body where microorganisms establish a symbiotic relationship with its host in complementing metabolic deficits, protection from invading pathogens and maintaining immune homeostasis (1). There is increasing evidence showing that gut microbiota influenced CRC development through the virulence factors of pathogenic bacterial strains, and recent reviews and papers have suggested a close correlation between certain microbial strains and CRC $(2,3,4,5,6)$. Bacteria such as Bacteroides fragilis, Fusobacterium nucleatum, and Escherichia coli have been identified to be enriched in patients with CRC $(7,8)$. Various molecular events as well as changes to the tumor microenvironment which are related to the enrichment of specific bacterial species were found to promote CRC tumorigenesis in various animal and ex vivo models $(9,10,11)$. These specific microbial strains associated with CRC provided promising opportunities to develop diagnostic tools or treatment biomarkers for CRC.

However, the relationship between the bacteriome and CRC appear complex as there is unlikely a single pathogenic bacteria that is related to $\mathrm{CRC}$, unlike the direct causal relationship that is established between Helicobacter pyloriand gastric cancer (12). Different microbial strains that are associated with CRC have been reported, some of which were not reproducible in other studies (13). For example, Dejea et al. found that there was no consistent bacterial genus associated with tumors by high-throughput sequencing in $30 \mathrm{CRC}$ and 6 adenoma human samples (13). In another study, the widely studied Fusobacterium that is increasingly associated with CRC was only found to be elevated in 20$30 \%$ of CRC patients and was not a consistent finding (14). On the other hand, other studies found differing microbes 
that triggered neoplasia and also proposed associated mechanisms. For example, Bacteroides fragilis was found to promote Th17 development which limits the availability of IL2 in the local microenvironment, while Escherichia coli releases colibactin which is a genomic product of polyketide synthase island that is carcinogenic and promotes CRC development $(6,15)$. Interacting with numerous microbes, the initiation and development of CRC appears to be fueled by a collective microbial dysbiosis rather than propelled by specific pathogenic microbes.

It is also inconclusive if the state of microbial dysbiosis associated with CRC continues to persist after curative surgery. Few studies have investigated the microbial alterations after curative CRC surgery. Kong et al. reported a reduced ratio of Bacteroidetes/ Firmicutes after surgery, which could contribute to intestinal inflammation (16). Tumorassociated microbes including Enterococcus and Fusobacterium were also found to be reduced. However, beneficial obligate anaerobes such as Prevotella were also reduced postoperatively. It is postulated that if the microbial dysbiosis is associated with the cancer, then resection of the tumour should not only remove the lesion but also reverse the dysbiosis that accompanied CRC. On the other hand, persistent dysbiosis after surgery may suggest possible field change in the "non-diseased" colon that predisposed the patient to CRC in the first place.

In this study, we thus seek to examine alterations in co-abundant bacterial strains in CRC patients before and after surgery and to also compare them to NC individuals. Using 16S rRNA gene sequencing, we first performed the classical community analysis and statistical tests to compare the gut microbial community structure and composition between CRC patients before (pre-op) and after surgery (post-op) and NC individuals. We then applied functional network analysis to further examine the differences among them.

\section{Results}

\section{Study population}

In this study, we have included a total of 37 individuals, of which 12 were CRC patients and 25 were NC controls. The participant and clinical data are summarized in Tables 1 and 2, respectively. Bacterial 16S rRNA gene sequencing of 49 fecal samples from CRC patients and NC controls were performed to investigate the compositional changes of the microbiome in CRC patients pre- and post-operatively (pre- and post-op). A total of 2,559,498 filtered sequences were obtained with an average of 52,234 sequences per sample (range $3,531-98,096$ ). A rarefaction curve for richness is plotted to analyse the sequencing depth of the samples (Supplementary Figure 1).

\section{Bacterial diversity is altered in CRC patients compared to NC individuals}

Firmicutes, Actinobacteria, Verrucomicrobia and Bacteroidetes are amongst the most abundant bacteria phyla found in all samples (Figure 1A), Global microbial alpha diversity was assessed using Chao1, ACE and Simpson across 3 groups. Chao1 and ACE indices were significantly decreased in post-operative patients $(p<0.05)$. In addition, an increase in Simpson index was observed for pre-op patients $(p<0.05)$ when compared to other groups in the analysis (Wilcoxon rank sum test, Figure 1B). Using principal coordinate analysis (PCoA) of the Bray-Curtis dissimilarity distances, beta diversity analysis of the groups showed that there is a clear segregation between CRC patients and NC (Figure 1C). Furthermore, microbial composition of post-op CRC patients presented a more scattered distribution, indicating a significantly different microbial composition as compared to pre-op CRC patients and NC. In all, the data suggest reduced fecal bacterial species richness and diversity in the CRC patients compared to the NC.

\section{Taxonomic differences in pre- and post-operative CRC patients compared to NC controls}


An overview of differentially abundant bacteria in CRC patients and NC fecal specimens was shown in Figure 2. Based on the heatmap representation of bacterial abundance, it is clear that there were significant alterations in the abundance of AVSs belonging to Actinobacteria, Firmicutes, Proteobacteria, Verrucomicrobia and Bacteroidetes phyla across the different groups. Of note, several specific ASVs belonging to Actinobacteria and Firmicutes were increased, whereas some Bacteroidetes AVSs were reduced in post-op CRC patients compared to preop patients.

Further detailed analysis revealed the level of changes in abundance of specific bacterial ASVs in pre- and post-op CRC patients compared to NC (Figure 3). As shown in Figure 3A, pre-op CRC patients have a higher abundance of several Bacteroidetes ASVs. Bacteroides caccae, Bacteroides vulgatus and Bacteroides fragilis were found to be increased in pre-op CRC patients ( $\mathbb{x} \leq 0.0001$, Figure $3 A$, pre-op column). Bacteria correlated with CRC prognosis, ASV 769 Odoribacter splanchnicus and bacteria correlated with a NC gut, ASV 694 Barnesiella intestinihominis, ASV 194 Anaerostipes hadrus, ASV 766 Blautia spp. and ASV 583 Eubacterium hallii group have more than 2 Log $_{2}$ fold increase in abundance in pre-op CRC (Figure 3B) $(21,22,23,24,25,26)$. However, these differences were no longer found in postop CRC compared to NC. Of note, the abundance of Bacteroides spp., B. caccae and B. vulgatus were reduced in postop CRC compared to pre-op based on paired analysis (Figure 3B). In contrast, fecal samples from pre-op patients have reduced ASV 308 Butyricimonas spp., ASV 515 Eubacterium coprostanoligenes group, ASV 285 Catenibacterium spp. and ASV 343 Lachnospiraceae FCS020 group, and these bacterial groups remained reduced even after post-op. Additionally, paired analysis revealed the influence of surgery on specific bacterial ASVs in CRC patients. These included the increase in ASV 269 Enterobacter spp., ASV 193 Klebsiella spp. and ASV 21 Akkermansia muciniphila.

\section{Microbial functional features associated with CRC development}

Gene content and functional pathways were inferred from the bacterial 16S rRNA gene sequencing data using Picrust2. The top differentially abundant KEGG (KOs) profiles from NC, pre- and post-op groups were compared using ANOVA Tukey-Kramer post-hoc comparison with Benjamini-Hochberg correction. PCoA plot (Figure 4A) showed that the KOs profiles from NC are significantly different from both pre- and post-op CRC (Benjamini-Hochberg FDR $<0.05$ ).

There were 66 and 300 differentially abundant KOs identified in pre- and post-op CRC compared to NC control group respectively ( $\mathrm{BH}$ adjusted $\mathrm{P} \leq 0.0015$, Supplementary Data 1 and 2 ). These KOs were mapped to KEGG pathways including metabolic pathways (23\% in pre-op, $34 \%$ in post-op); biosynthesis of secondary metabolites ( $10 \%$ in both pre-op and post-op) and microbial metabolism in diverse environments ( $3 \%$ in pre-op, $8 \%$ in post-op). The differentially abundant KOs were also assigned to BRITE hierarchies. The most represented BRITE hierarchies included; enzymes (51\% in pre-op, $34 \%$ in post-op), two-component system (6\% in pre-op, $3 \%$ in post-op), prokaryotic defense system (5\% in pre-op, $1 \%$ in post-op) and transcription machinery ( $5 \%$ in pre-op, $1 \%$ in post-op).

Interestingly, KOs including K00392 sir; sulfite reductase (ferredoxin) was found to be significantly reduced in pre- and post-op (-5.3 and $-6.3 \log _{2}$ fold change respectively, adj. P $\leq 0.0001$ ) compared to NC (Figures 4B and 4C), which mapped to the MODULE "M00176 Assimilatory sulfate reduction, sulfate => H2S". The importance of the altered sulfur metabolism was also identified in subsequent detailed pathway enrichment analysis using clusterProfiler $\mathrm{R}$ package (27). "Sulfur metabolism" was significantly lower in pre-op CRC compared to NC controls (Figure 5A and

Supplementary Figure 2). K00392 is highly associated with pathways including "Sulfur metabolism" and "Microbial metabolism in diverse environments", indicating a buildup of sulfate in the colonic environment due to the inability to metabolize sulfate to sulfur amino acids. ${ }^{28,29}$ Additionally, $A B C$ transporters remain over-presented in post-op (when compared to either NC or pre-op), suggesting that surgical intervention did not manage to change the bacteria group 
that carry out this function in the patients. The key KOs in ABC transporter are; trehalose/maltose transport system, cellobiose transport system, urea transport system, fluoroquinolone transport system (Supplementary Data 1 and 2 ).

Of note, enrichment analysis showed that Lipoarabinomannan (LAM) biosynthesis was suppressed in post-op compared to NC control group. Lipoarabinomannan is a virulence factor commonly associated with Mycobacteria tuberculosis infection. It is also expressed by many other Actinomycetes bacteria including Gordonia, Rhodococcus, Tsukamurella, Amycolatopsis and Corynebacterium. While the sequencing data did not have sufficient resolution to identify each of these Actinomycetes bacteria (M. tuberculosis was not detected), we found that the suppression of (LAM) biosynthesis was due to reduced abundance of specific KOs (Supplementary Figure 2) and ASVs belonging to the Actinomycetaceae family, in particular ASV581 and ASV1102.

K19076 cmr2, cas10; CRISPR-associated protein Cmr2, K19141 cmr5; CRISPR-associated protein Cmr5 and K14440 SMARCAL1, HARP were more abundant (3 $\log _{2}$ fold) in pre-op patients compared to NC. These KOs are highly associated with BRITE functions including "Prokaryotic defense system" and "Chromosome and associated proteins"

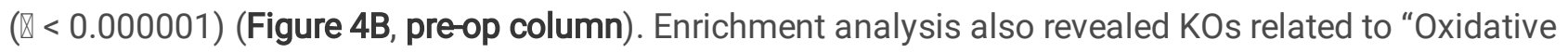
phosphorylation", "Thermogenesis" and "Valine, leucine \& isoleucine degradation" were suppressed in post-op CRC patients $(\mathbb{\nabla}=0.000001)$ (Figure 5B and Supplementary Figure 2).

When comparing post-op to NC, K08973 hemJ; protoporphyrinogen IX oxidase was reduced by 4 Log $_{2}$ fold. This KO is highly associated with "Heme biosynthesis, plants and bacteria, glutamate". Studies have demonstrated that glutamine stimulates the expression of $\mathrm{HO}-1$, a protein that is involved in maintaining intestinal cell proliferation and repair (30). With the presence of pathogenic bacteria such as Enterobacter spp., Klebsiella spp., and Hungatella hathewayi (Figure 3B) and with a compromised immune function, this could indicate the colonic environment remains in a state of dysbiosis and is prone to damage after surgery.

Interestingly, thermogenesis is suppressed in post-op CRC patients. Studies have reported that amino acids valine, leucine and isoleucine participate in lipogenesis, lipolysis and immune function of the gut (31). Lipogenesis and lipolysis can be circumvented through supplementation of microbial butyrate. However, butyrate producing bacteria, Lachnospiraceae, was observed to have diminished (Figure 3B) thus subjecting the gut environment to a compromised adaptive thermogenesis. ${ }^{32}$ Similarly, reduction in K02274 coxA, ctaD; cytochrome c oxidase subunit I and K02276 coxC, ctaE; cytochrome c oxidase subunit III, which are associated with "Oxidative phosphorylation", were also observed. Studies have demonstrated that mitochondrial mutations affect downstream processes of the electron transport chain system, which indirectly contribute to an increase in production of reactive oxygen species leading to a compromised repair system $(33,34)$.

Further analysis to evaluate the influence of surgery on microbial dynamics were carried out by comparing the changes in predicted function profile between pre- and post-op using paired sample test. A total of 25 differentially abundant KOs were identified (BH adjusted $\mathrm{P} \leq 0.0015$, Supplementary Data 3 ). These KOs were mapped to KEGG pathways including metabolic pathways (28\%); ABC transporters (8\%); Carbon metabolism (8\%). Interestingly, genes associated with PATHWAY function "ABC transporters", K13890 gsiC; glutathione transport system permease protein and K17315 gtsA; glucose/mannose transport system substrate-binding protein, were found to be over-represented in post-op, suggesting that surgical intervention leads to increase bacteria that may be responsible for this function in CRC patients (Supplementary Data 1, 2 and 3). Enrichment analysis revealed genes related to "cell cycle - Caulobacter" and "metabolic pathways" were suppressed in post-op CRC patients when compared to their pre-op state $(\mathbb{Z}=0.001)$ (Figure 5C and Supplementary Figure 2) as demonstrated with a $4 \log _{2}$ fold reduced expression of K13581 ccrM; modification methylase, K01771 plc; 1-phosphatidylinositol phosphodiesterase, K05304 NANS, SAS; sialic acid 
synthase and K16649 glft1 (Figure 4C). With a compromised repair system and energy metabolism, links to a faulty cell cycle of the gut microbiome could be established (35).

\section{Discussion}

The adenoma-carcinoma sequence is a well-known theory describing the genetic pathogenesis of colorectal cancer (36). Initial genetic changes through pathways such as chromosomal instability, microsatellite instability and CpG island methylator phenotype pathways, start in an early adenoma and accumulate as it transforms to carcinoma (37). However, when considering the genetics of CRC, only $10 \%$ of cases are inherited and only $2-6 \%$ can be ascribed to highly penetrant genes, such as APC, hMLH and hMSH2 (38). The vast majority of CRC cases are sporadic and noninherited. A Western diet, smoking, obesity, alcohol consumption and exposure to carcinogens have been validated as important risk factors for the development of sporadic CRC (39). Although there has been long-standing associations between diet, microbiome and $\mathrm{CRC}$, only recently have we begun to further explore the associations between gut microbiome and CRC (40). The role of microbiome may represent one missing piece to explain the susceptibility of certain patients to CRC. In the past decade, multiple reviews and studies have analysed the relationship between specific microbes, such as Fusobacteria and B. fragilis, and CRC $(36,37,38,39,40)$. However, evidence behind their direct associations remain conflicting and there is unlikely a single pathogen related to the development of CRC, unlike the established causal relationship between H. pylori and gastric cancer, or Epstein-Barr Virus and nasopharyngeal cancer $(36,37,38,49,40,41)$. Combinations or co-abundance groups of organisms seem to be operative in CRC, rather than representing a one organism-one disease model.

Our study found obvious differences between CRC patients and NC individuals. There was reduced fecal bacterial species richness and diversity in the CRC patients compared to the NC control group (Figure 1), which is consistent with previous studies reporting that high diversity is associated with health and temporal stability, while a relative lack of diversity is often observed in the microbiota of CRC patients (42,43). Pre-op CRC patients have a significantly higher abundance of several Bacteroidetes ASVs. B. caccae and B. vulgatus are known to be involved in destabilizing the colonic wall of the gut, potentially resulting in progression of CRC. ${ }^{44,45}$ In addition, Odoribacter splanchnicus was identified to be significantly increased while probiotic bacteria Blautia spp. and Romboutsia spp. were reduced in preop CRC patients. Odoribacter are correlated to somatic mutations and cell proliferation resulting in poorer CRC prognosis while Blautia spp. and Romboutisa spp. are associated with a NC gut $(22,26)$.

Interestingly, these differences were no longer observed after surgery when compared to NC individuals. At the same time, there was an increased abundance of probiotic bacteria such as Bifidobacterium spp. (Bifidobacterium dentium and Bifidobacterium breve), Lactobacillus spp, Akkermansia muciniphila, Anaerostipes caccae and Colidextribacter spp. after surgery (paired analysis between post- and pre-op, Figure $3 \mathbf{B}$ ). These probiotics have been demonstrated to fortify the colonic mucus layer, competitively exclude pathogenic bacteria and regulate the colonic environment through regulating of immune cells for anti-tumour activity $(46,47,48,49,50,51)$. The increased abundance of beneficial gut genera after surgery such as short-chain fatty acid (SCFA) producing microbiota (Roseburia spp. and Blautia spp.) and gut barrier enhancer (Lactobacillus spp.) suggest that surgery partially "reverts" the gut back to its "NC" precancerous state to a certain degree. ${ }^{26,51}$

However, presence of pathogenic bacteria such as Enterobacter spp., Klebsiella spp. and Hungatella hathewayi could indicate that the colonic environment is still in a state of dysbiosis as these bacteria are known to be oncogenic $(52,53,54)$. Microbiota associated with CRC progression such as Clostridium innocuum, Eubacterium brachy, Eggerthella spp. were also observed to be significantly abundant in post-operative CRC patients $(55,56,57)$. This illustrates a possible field-change in microbial composition in the colon of CRC patients, where there is persistence of 
oncogenic microbial species even after surgery. Future longitudinal studies can seek to examine if microbial dysbiosis persists in the remnant "normal" colonic tissue in patients after CRC surgery and its significance.

Our study found that curative surgery resulted in the reversion of some bacterial strains (i.e., Bacteroidetes spp.) to a "non-cancer" level but not others (i.e., Eubacterium coprostanoligenes group). On the other hand, certain bacterial groups which were found in equal abundance in NC patients were increased after surgery (i.e., A. muciniphila.). These suggest a complex dynamics of bacteriome field-change that is associated with the presence of CRC. CRC associated microbiome signatures likely comprise a set of associated microbial and tumor microenvironment changes which may be more easily identified with artificial intelligence and deep machine learning platforms (58). Instead of investigating single microbial strains that are believed to be oncogenic, the focus of future studies should perhaps be on multi-omic techniques and machine learning to simplify large microbiome datasets into biologically relevant information so as to identify microbial and related metabolite patterns that are characteristic of CRC. This can help to validate the microbial signatures which are found in our study, which comprise both significant increases and reductions in specific bacterial strains, which when taken together, represents the unique microbial dysbiosis of CRC.

Besides utilising microbial strains which are found in greater abundance in CRC, equal emphasis should be given to other significant strains that are reduced after surgery. The microbial dysbiotic signatures associated with CRC will therefore comprise a specific pattern of alteration in bacteriome. This contrasts with the existing trend of simply identifying specific microbial strains as a complementary tool for the screening and diagnosis of CRC as proposed by various studies. Moreover, these specific patterns can also be used to screen for disease recurrence in CRC patients after surgery, where the return of specific microbial markers can indicate recurrent disease. A follow-up study on the current cohort can seek to examine possible changes in bacteriome in association with disease remission and recurrence.

More importantly, it will be interesting to examine if a similar pattern of microbial dysbiosis is present in patients with adenomatous polyps, which are precursors of $\mathrm{CRC}$, and if these signatures are lost after polypectomy is performed. Studies have reported specific microbes associated with the presence of colonic polyps, which supports the presence of a unique microbial profile even in patients with pre-malignant polyps $(59,60)$. If found, these microbial dysbiotic signatures may perhaps have the potential to be used as a predictor for patients with polyps and who thus require more urgent colonoscopy. This can help streamline CRC screening and has the potential to guide the allocation and prioritization of colonoscopy, which is costly and not without risks. It may even help guide the indication for surveillance colonoscopy by identifying patients with a greater chance of having recurrent or persistent polyps.

Our study has certain limitations. First, microbial analysis was based on stool samples, which is postulated to reflect the cancer microbiota due to the downstream shedding of cancer cells. However, it might not fully represent the altered microbial profile of the cancer tissue and we are also unable to study changes in the tumor microenvironment. Second, 16S rRNA sequencing is a widely used metagenomic approach in understanding the gut microbiome but has insufficient resolution to the level of microbial strains, and also does not provide details on the presence of specific pathogenic metabolism related genes that can improve the characterization of functional landscape. Therefore, associations that are derived will be less specific and shotgun sequencing can improve the confidence in predicting functional gene profile. Last but not least, this is a pilot study with a small sample size, and the recruited CRC patients all had left-sided colon cancer incidentally. As right and left-sided colon cancers are known to have different molecular biological characteristics and possibly differentially expressed microbial species, the results of our study may not be applicable to right-sided colon cancers (61).

\section{Conclusion}


Microbiome signatures characteristic of CRC likely involves a set of associated microbial alterations which comprise both significant increases and reductions in specific bacterial strains. Elements of this unique microbial dysbiosis of CRC persists even after surgery, suggesting possible field-change in the remnant non-diseased colon. Future studies should seek examine if these dysbiotic patterns are also associated with pre-malignant colonic polyps and explore if the unique microbial profiles can be used to guide CRC screening and surveillance.

\section{Methods}

\section{Study participants and fecal sample collection}

Ethics approval to conduct this study was obtained by the National Healthcare Group domain specific review board (NHG-DSRB), reference number: 2017/01257. All methods were carried out in accordance with relevant guidelines and regulations as outlined by National Healthcare Group domain specific review board, Singapore.

All patients aged 21 and above who were scheduled to undergo elective diagnostic colonoscopy between May 2018 to November 2018 were invited to participate in this study. Informed consent was obtained from all participants and or their legal guardian(s) prior their participation to the study. Participants who were found to have CRC at colonoscopy were assigned to the "Cancer" group, while those who did not have cancer were assigned to the "Non-cancer" group. Exclusion criteria were patients who were unable to provide written consent for participation in the study, had preexisting family history of familial adenomatous polypopsis (FAP) or hereditary non-polypopsis colorectal cancer (HNPCC), diagnosed inflammatory bowel disease and/ or consumption of antibiotics during the three months prior to the study.

To compare changes in the gut microbiome profiles of CRC patients before and after surgery, fecal samples at 2 sampling time points were collected from the cancer group. The 2 points were 1 . Prior to colonoscopy (pre-op), and 2. Six months after surgery (post-op). On the other hand, only one fecal sample prior to colonoscopy was collected from each individual in the Non-cancer group (NC). As there have been reports of microbial alterations after mechanical bowel preparation, the stool samples were taken before consumption of polyethylene glycol. A total of 49 fecal samples were collected using sterile fecal collection tubes containing 4 sterile glass beads measuring $5 \mathrm{~mm}$ in diameter (Merck, Germany) and $2 \mathrm{ml}$ of RNAlater ${ }^{\circledR}$ (Invitrogen, Lithuania). Samples were stored at $-80^{\circ} \mathrm{C}$ prior to downstream processes.

\section{Bacterial 16S rRNA gene sequencing}

Fecal samples were thawed and vortexed to produce a homogenate and washed once using $1 \mathrm{X}$ phosphate buffer saline (Vivantis Technologies, Malaysia). Genomic DNA was extracted using DNeasy PowerSoil Pro Kit (Qiagen, Germany) according to manufacturers' protocol. The purity and DNA yield for each fecal sample was measured using a nanodrop.

In this study, the V3-V4 region of 16S rRNA was sequenced to analyse composition of the gut microbiota of fecal samples. A PCR targeting the V3-V4 region using forward (5'-CCTACGGGNGGCWGCAG) and reverse primers (5'GACTACHVGGGTATCTAATCC) with overhang adaptors, were performed as recommended by the 16S metagenomics library kit by Illumina. The quality and quantity of the amplicons were measured using Agilent 4200 TapeStation, picogreen and nanodrop. All samples passed the quality control measurement and proceeded for a second round of PCR step for library preparation. Library qualities were measured using Agilent 4200 TapeStation, picogreen and qPCR. Libraries that passed the quality control measurement were pooled as recommended by Illumina and were sequenced using the MiSeq platform using 2 x 300 PE format. 
Sequence reads were deposited into the NCBI Sequence read archive (SRA) as part of BioProject number

PRJNA662014.

\section{Microbiome profiling}

Raw fastq files were quality filtered, merged, demultiplexed and denoised using DADA2 (version 1.14.1) microbiome pipeline with default parameters (17). Output from DADA2, known as amplicon sequence variants (ASVs), were cleaned up by removing chimeras. ASVs is a clustering method for better precision and improve resolution of sequence identity. ASVs were then assigned to their respective taxonomy using Silva (version 138) classifier.

Statistical analysis was carried out in $\mathrm{R}$ (version 3.6.3) and analysis of microbiome diversities was carried out using the Phyloseq package (version 1.30.0). Alpha diversity was calculated using Chao1, ACE and Simpson. Diversity differences between CRC (at each sampling time point) and NC groups were analysed using Wilcoxon rank sum test. Inter-group similarities were visualized using principal coordinate analysis (PCoA) of the Bray-Curtis dissimilarity distances. The DESeq2 package (version 1.26.0) was used to identify microbiota that showed significant differential abundances across NC, pre-op and post-op, while adjusted for confounders such as gender and age. Predicted functional information from the gut microbiome data was generated using PICRUSt2 (version 2.3.0) pipeline with default parameters $(18,19,20)$.

\section{Declarations}

\section{Acknowledgments}

This work was supported by the National University Health System Seed Fund [NUHSRO/2020/142/RO5+6/SeedSep/02]; and the The Lee Foundation.

Author contributions:

(I) Conception and design: CWP, YKC, JHL, KKT

(II) Administrative support: CWP, YKC, YZ, KKT

(III) Provision of study materials or patients: YKC, KKT

(IV) Collection and assembly of data: CWP, YKC

(V) Data analysis and interpretation: CWP, YKC, JHL

(VI) Manuscript writing: All authors

(VII) Final approval of manuscript: All authors

Conflicts of Interest:

The authors have no conflicts of interest to declare.

\section{References}

1. Neish AS. Microbes in gastrointestinal health and disease. Gastroenterology. 2009;136(1):65-80.

2. Marchesi JR, Dutilh BE, Hall N, Peters WHM, Roelofs R, Boleij A, et al. Towards the Human Colorectal Cancer Microbiome. PLOS ONE. 2011;6(5):e20447.

3. Marchesi JR, Adams DH, Fava F, Hermes GD, Hirschfield GM, Hold G, Quraishi MN, Kinross J, Smidt H, Tuohy KM et al. 2016. The gut microbiota and host health: A new clinical frontier. Gut. 65(2):330-339. 
4. Scanlan PD, Shanahan F, Clune Y, Collins JK, O'Sullivan GC, O'Riordan M, et al. Culture-independent analysis of the gut microbiota in colorectal cancer and polyposis. Environ Microbiol. 2008;10(3):789-98.

5. Ou J, Carbonero F, Zoetendal EG, DeLany JP, Wang M, Newton K, et al. Diet, microbiota, and microbial metabolites in colon cancer risk in rural Africans and African Americans. Am J Clin Nutr. 2013;98(1):111-20.

6. Arthur JC, Perez-Chanona E, Mühlbauer M, Tomkovich S, Uronis JM, Fan TJ, et al. Intestinal inflammation targets cancer-inducing activity of the microbiota. Science. 2012;338(6103):120-3.

7. Dejea CM, Fathi P, Craig JM, Boleij A, Taddese R, Geis AL, et al. Patients with familial adenomatous polyposis harbor colonic biofilms containing tumorigenic bacteria. Science. 2018;359(6375):592-7.

8. Kostic AD, Gevers D, Pedamallu CS, Michaud M, Duke F, Earl AM, et al. Genomic analysis identifies association of Fusobacterium with colorectal carcinoma. Genome Res. 2012;22(2):292-8.

9. Yang Y, Weng W, Peng J, Hong L, Yang L, Toiyama Y, et al. Fusobacterium nucleatum Increases Proliferation of Colorectal Cancer Cells and Tumor Development in Mice by Activating Toll-Like Receptor 4 Signaling to Nuclear FactorKB, and Up-regulating Expression of MicroRNA-21. Gastroenterology. 2017;152(4):851-66.e24.

10. Kostic AD, Chun E, Robertson L, Glickman JN, Gallini CA, Michaud M, et al. Fusobacterium nucleatum potentiates intestinal tumorigenesis and modulates the tumor-immune microenvironment. Cell Host Microbe. 2013;14(2):207-15.

11. Dharmani P, Strauss J, Ambrose C, Allen-Vercoe E, Chadee K. Fusobacterium nucleatum infection of colonic cells stimulates MUC2 mucin and tumor necrosis factor alpha. Infect Immun. 2011;79(7):2597-607.

12. Ishaq S, Nunn L. Helicobacter pylori and gastric cancer: a state of the art review. Gastroenterol Hepatol Bed Bench. 2015;8(Suppl 1):S6-s14.

13. Dejea CM, Wick EC, Hechenbleikner EM, White JR, Mark Welch JL, Rossetti BJ, et al. Microbiota organization is a distinct feature of proximal colorectal cancers. Proc Natl Acad Sci U S A. 2014;111(51):18321-6.

14. Flemer B, Herlihy M, O'Riordain M, Shanahan F, O'Toole PW. Tumour-associated and non-tumour-associated microbiota: Addendum. Gut Microbes. 2018;9(4):369-73.

15. Geis AL, Fan H, Wu X, Wu S, Huso DL, Wolfe JL, et al. Regulatory T-cell Response to Enterotoxigenic Bacteroides fragilis Colonization Triggers IL17-Dependent Colon Carcinogenesis. Cancer Discov. 2015;5(10):1098-109.

16. Kong C, Gao R, Yan X, Huang L, He J, Li H, et al. Alterations in intestinal microbiota of colorectal cancer patients receiving radical surgery combined with adjuvant CapeOx therapy. Sci China Life Sci. 2019;62(9):1178-93.

17. Callahan BJ, McMurdie PJ, Rosen MJ, Han AW, Johnson AJ, Holmes SP. DADA2: High-resolution sample inference from Illumina amplicon data. Nat Methods. 2016;13(7):581-3.

18. Douglas GM, Maffei VJ, Zaneveld J, Yurgel SN, Brown JR, Taylor CM, et al. PICRUSt2: An improved and extensible approach for metagenome inference. bioRxiv. 2019:672295.

19. Ye Y, Doak TG. A Parsimony Approach to Biological Pathway Reconstruction/Inference for Genomes and Metagenomes. PLOS Computational Biology. 2009;5(8):e1000465. 
20. Douglas GM, Maffei VJ, Zaneveld JR, Yurgel SN, Brown JR, Taylor CM, et al. PICRUSt2 for prediction of metagenome functions. Nature Biotechnology. 2020;38(6):685-8.

21. Yazici C, Wolf PG, Kim H, Cross T-WL, Vermillion K, Carroll T, et al. Race-dependent association of sulfidogenic bacteria with colorectal cancer. Gut. 2017;66(11):1983-94.

22. Cheng WY, Wu C-Y, Yu J. The role of gut microbiota in cancer treatment: friend or foe? Gut. 2020;69(10):1867.

23. Ai D, Pan H, Li X, Gao Y, Liu G, Xia LC. Identifying Gut Microbiota Associated With Colorectal Cancer Using a Zero-Inflated Lognormal Model. Frontiers in Microbiology. 2019;10(826).

24. Wu Y, Jiao N, Zhu R, Zhang Y, Wu D, Wang A-J, et al. Identification of microbial markers across populations in early detection of colorectal cancer. Nature Communications. 2021;12(1):3063.

25. Liu X, Mao B, Gu J, Wu J, Cui S, Wang G, et al. Blautia-a new functional genus with potential probiotic properties? Gut Microbes. 2021;13(1):1-21.

26. Engels C, Ruscheweyh H-J, Beerenwinkel N, Lacroix C, Schwab C. The Common Gut Microbe Eubacterium hallii also Contributes to Intestinal Propionate Formation. Frontiers in Microbiology. 2016;7(713).

27. Li N, Huang S, Jiang L, Dai Z, Li T, Han D, et al. Characterization of the Early Life Microbiota Development and Predominant Lactobacillus Species at Distinct Gut Segments of Low- and Normal-Birth-Weight Piglets. Frontiers in Microbiology. 2019;10(797).

28. Yu G, Wang LG, Han Y, He QY. clusterProfiler: an R package for comparing biological themes among gene clusters. Omics. 2012;16(5):284-7.

29. Dordević D, Jančíková S, Vítězová M, Kushkevych I. Hydrogen sulfide toxicity in the gut environment: Metaanalysis of sulfate-reducing and lactic acid bacteria in inflammatory processes. Journal of Advanced Research. 2021;27:55-69.

30. Kushkevych I, Cejnar J, Treml J, Dordević D, Kollar P, Vítězová M. Recent Advances in Metabolic Pathways of Sulfate Reduction in Intestinal Bacteria. Cells. 2020;9(3):698.

31. Oates P-S, West A-R. Heme in intestinal epithelial cell turnover, differentiation, detoxification, inflammation, carcinogenesis, absorption and motility. World J Gastroenterol. 2006;12(27):4281-95.

32. Zhang S, Zeng X, Ren M, Mao X, Qiao S. Novel metabolic and physiological functions of branched chain amino acids: a review. J Anim Sci Biotechnol. 2017;8:10-.

33. Li B, Li L, Li M, Lam SM, Wang G, Wu Y, et al. Microbiota Depletion Impairs Thermogenesis of Brown Adipose Tissue and Browning of White Adipose Tissue. Cell Rep. 2019;26(10):2720-37.e5.

34. Ashton TM, McKenna WG, Kunz-Schughart LA, Higgins GS. Oxidative Phosphorylation as an Emerging Target in Cancer Therapy. Clinical Cancer Research. 2018;24(11):2482.

35. Namslauer I, Brzezinski P. A mitochondrial DNA mutation linked to colon cancer results in proton leaks in cytochrome \&lt;em\&gt;c\&lt;/em\&gt; oxidase. Proceedings of the National Academy of Sciences. 2009;106(9):3402. 
36. Gao P, Ma C, Sun Z, Wang L, Huang S, Su X, et al. Feed-additive probiotics accelerate yet antibiotics delay intestinal microbiota maturation in broiler chicken. Microbiome. 2017;5(1):91.

37. Leslie A, Carey FA, Pratt NR, Steele RJ. The colorectal adenoma-carcinoma sequence. Br J Surg. 2002;89(7):845-60.

38. Armaghany T, Wilson JD, Chu Q, Mills G. Genetic alterations in colorectal cancer. Gastrointest Cancer Res. 2012;5(1):19-27.

39. Bogaert J, Prenen H. Molecular genetics of colorectal cancer. Ann Gastroenterol. 2014;27(1):9-14.

40. Carr PR, Alwers E, Bienert S, Weberpals J, Kloor M, Brenner H, et al. Lifestyle factors and risk of sporadic colorectal cancer by microsatellite instability status: a systematic review and meta-analyses. Ann Oncol. 2018;29(4):825-34.

41. Aries V, Crowther JS, Drasar BS, Hill MJ, Williams RE. Bacteria and the aetiology of cancer of the large bowel. Gut. 1969;10(5):334-5.

42. Young LS, Dawson CW. Epstein-Barr virus and nasopharyngeal carcinoma. Chin J Cancer. 2014;33(12):581-90.

43. Flores GE, Caporaso JG, Henley JB, Rideout JR, Domogala D, Chase J, et al. Temporal variability is a personalized feature of the human microbiome. Genome Biology. 2014;15(12):531.

44. Gao Z, Guo B, Gao R, Zhu Q, Qin H. Microbiota disbiosis is associated with colorectal cancer. Frontiers in Microbiology. 2015;6(20).

45. Sánchez-Alcoholado L, Ordóñez R, Otero A, Plaza-Andrade I, Laborda-Illanes A, Medina JA, et al. Gut Microbiota-Mediated Inflammation and Gut Permeability in Patients with Obesity and Colorectal Cancer. Int J Mol Sci. 2020;21(18):6782.

46. Feng Q, Liang S, Jia H, Stadlmayr A, Tang L, Lan Z, et al. Gut microbiome development along the colorectal adenoma-carcinoma sequence. Nature Communications. 2015;6(1):6528.

47. Engevik MA, Luk B, Chang-Graham AL, Hall A, Herrmann B, Ruan W, et al. Bifidobacterium dentium Fortifies the Intestinal Mucus Layer via Autophagy and Calcium Signaling Pathways. mBio. 2019;10(3):e01087-19.

48. Wei H, Chen L, Lian G, Yang J, Li F, Zou Y, et al. Antitumor mechanisms of bifidobacteria. Oncol Lett. 2018;16(1):3-8.

49. Zhong L, Zhang X, Covasa M. Emerging roles of lactic acid bacteria in protection against colorectal cancer. World J Gastroenterol. 2014;20(24):7878-86.

50. Dutta D, Lim SH. Bidirectional interaction between intestinal microbiome and cancer: opportunities for therapeutic interventions. Biomarker Research. 2020;8(1):31.

51. Tarashi S, Siadat SD, Ahmadi Badi S, Zali M, Biassoni R, Ponzoni M, et al. Gut Bacteria and their Metabolites: Which One Is the Defendant for Colorectal Cancer? Microorganisms. 2019;7(11):561.

52. Mager LF, Burkhard R, Pett N, Cooke NCA, Brown K, Ramay H, et al. Microbiome-derived inosine modulates response to checkpoint inhibitor immunotherapy. Science. 2020;369(6510):1481. 
53. Yurdakul D, Yazgan-Karataş A, Şahin F. Enterobacter Strains Might Promote Colon Cancer. Curr Microbiol. 2015;71(3):403-11.

54. Lai HC, Lin CC, Cheng KS, Kao JT, Chou JW, Peng CY, et al. Increased incidence of gastrointestinal cancers among patients with pyogenic liver abscess: a population-based cohort study. Gastroenterology. 2014;146(1):12937.e1.

55. Xia X, Wu WKK, Wong SH, Liu D, Kwong TNY, Nakatsu G, et al. Bacteria pathogens drive host colonic epithelial cell promoter hypermethylation of tumor suppressor genes in colorectal cancer. Microbiome. 2020;8(1):108.

56. Chia JH, Feng Y, Su LH, Wu TL, Chen CL, Liang YH, et al. Clostridium innocuum is a significant vancomycinresistant pathogen for extraintestinal clostridial infection. Clinical Microbiology and Infection. 2017;23(8):560-6.

57. Lazar V, Ditu L-M, Pircalabioru GG, Gheorghe I, Curutiu C, Holban AM, et al. Aspects of Gut Microbiota and Immune System Interactions in Infectious Diseases, Immunopathology, and Cancer. Frontiers in Immunology. 2018;9(1830).

58. Sheng Q, Du H, Cheng X, Cheng X, Tang Y, Pan L, et al. Characteristics of fecal gut microbiota in patients with colorectal cancer at different stages and different sites. Oncol Lett. 2019;18(5):4834-44.

59. Zeng T, Yu X, Chen Z. Applying artificial intelligence in the microbiome for gastrointestinal diseases: A review. J Gastroenterol Hepatol. 2021;36(4):832-40.

60. Sobhani I, Amiot A, Le Baleur Y, Levy M, Auriault M-L, Van Nhieu JT, et al. Microbial dysbiosis and colon carcinogenesis: could colon cancer be considered a bacteria-related disease? Therap Adv Gastroenterol. 2013;6(3):215-29.

61. Dadkhah E, Sikaroodi M, Korman L, Hardi R, Baybick J, Hanzel D, et al. Gut microbiome identifies risk for colorectal polyps. BMJ Open Gastroenterol. 2019;6(1):e000297-e.

\section{Tables}

Table 1 I Overview of participant clinical characteristics

\begin{tabular}{|c|c|c|}
\hline & Cancer $(\%$ or SD) & Healthy (\% or SD) \\
\hline & $\mathrm{n}=12$ & $\mathrm{n}=25$ \\
\hline Age, mean & $63.8( \pm 9.3)$ & $61.6( \pm 8.9)$ \\
\hline \multicolumn{3}{|l|}{ Gender } \\
\hline Male & $7(58.3 \%)$ & $14(56.0 \%)$ \\
\hline Female & $5(41.7 \%)$ & $11(44.0 \%)$ \\
\hline Body mass index, mean & $25.8( \pm 4.0)$ & $25.2( \pm 4.4)$ \\
\hline Adjuvant chemotherapy & $7(58.3 \%)$ & $\nabla$ \\
\hline
\end{tabular}

Table 2 I Clinical data of cancer group patients 


\begin{tabular}{|llllll|}
\hline Subject & Age & Gender & Race & Colonic tumor location & TNM \\
& & & & & Cancer stage \\
M02-P & 57 & Male & Malay & Sigmoid & 3 \\
M03-P & 74 & Female & Chinese & Descending & 3 \\
\hline M04-P & 56 & Female & Malay & Sigmoid & 1 \\
\hline M06-P & 60 & Female & Malay & Sigmoid & 3 \\
\hline M07-P & 67 & Male & Chinese & Sigmoid & 2 \\
\hline M08-P & 46 & Male & Chinese & Rectum & 3 \\
\hline M09-P & 59 & Female & Indian & Rectum & 2 \\
\hline M11-P & 65 & Male & Chinese & Sigmoid & 2 \\
\hline M12-P & 65 & Male & Indian & Rectosigmoid & 1 \\
\hline M13-P & 69 & Male & Chinese & Sigmoid & 3 \\
\hline M15-P & 82 & Female & Chinese & Rectosigmoid & 3 \\
\hline M16-P & 66 & Male & Chinese & Sigmoid & 1 \\
\hline
\end{tabular}

\section{Figures}
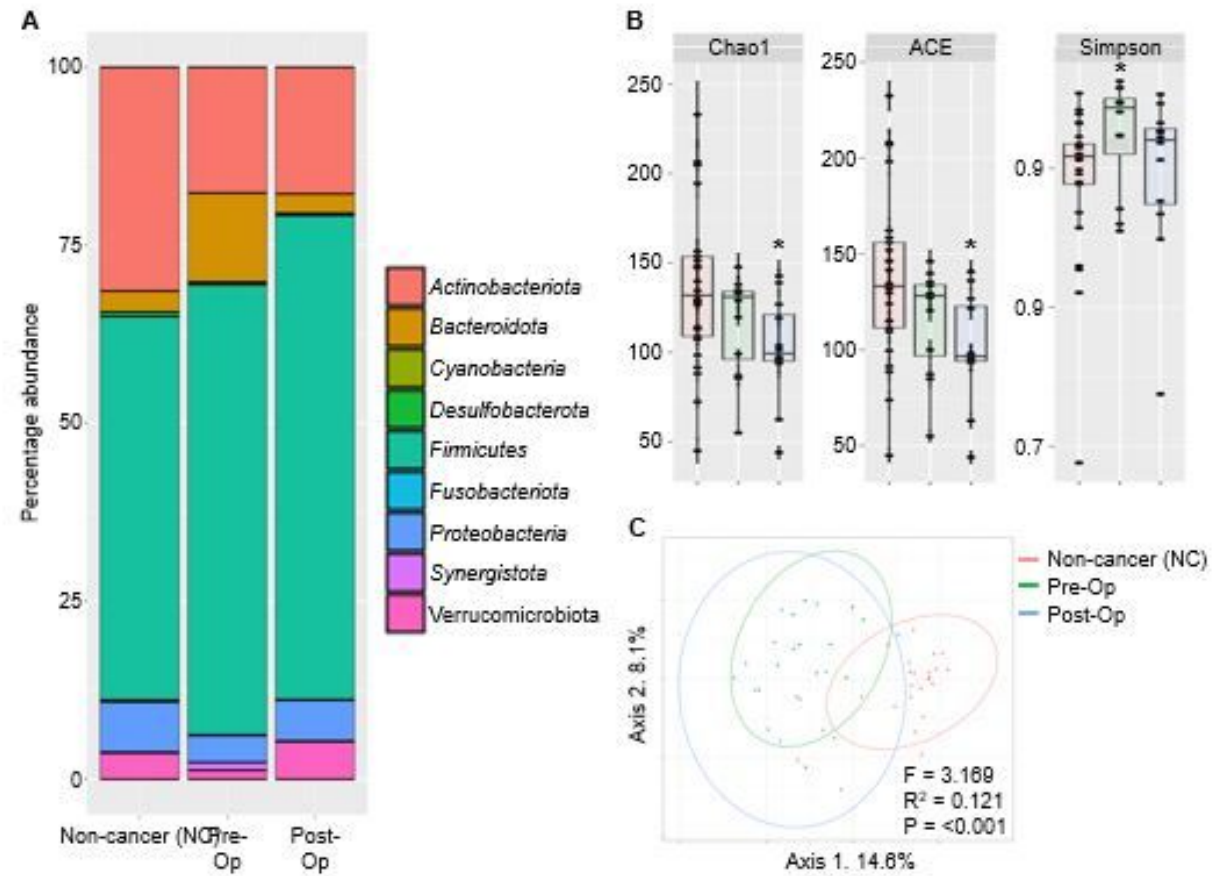

Figure 1

$<p>$ Fecal bacterial compositions are different between NC and CRC patients. (A) Bar chart shows the top bacterial phyla present in all samples. (B) Comparisons of bacterial alpha diversity in CRC to NC control group. * denotes $p$ \&lt; 
0.05 (Wilcoxon rank sum test). (C) Principal coordinates analysis (PCoA) of bacterial beta diversity derived from BrayCurtis distances among specimens is shown. F statistics, $R<$ sup $>2</$ sup $>$ \&amp; $p$ values of the comparison are presented (PERMANOVA) $.</ p><p><b r></ p>$

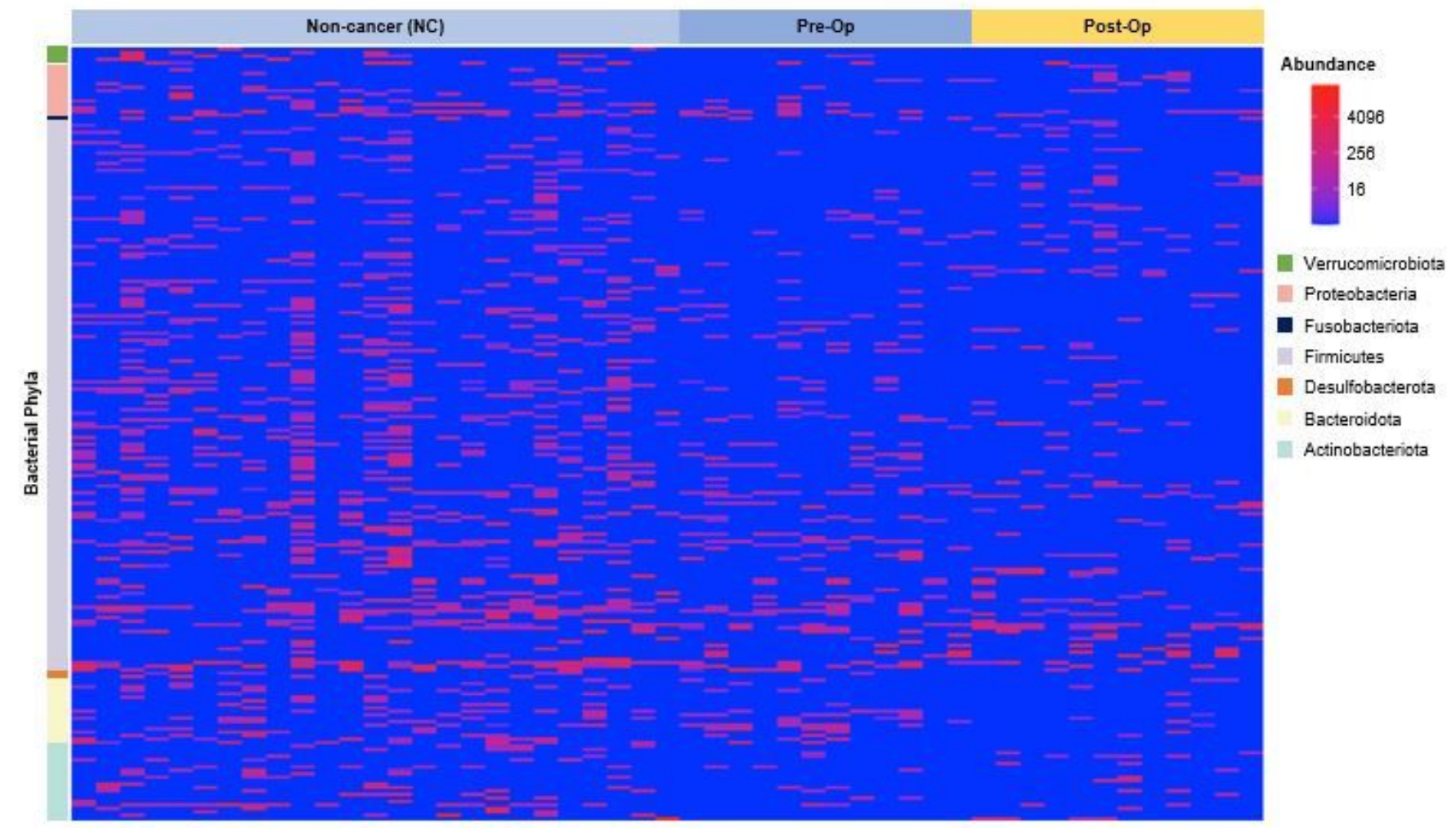

\section{Figure 2}

$<p>$ Heatmap showing differentially abundant bacterial phyla across NC and CRC patients. Top differentially abundant

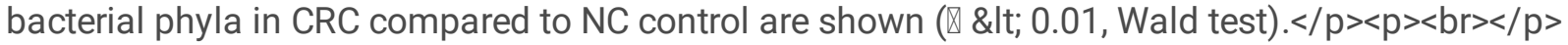




\section{A}

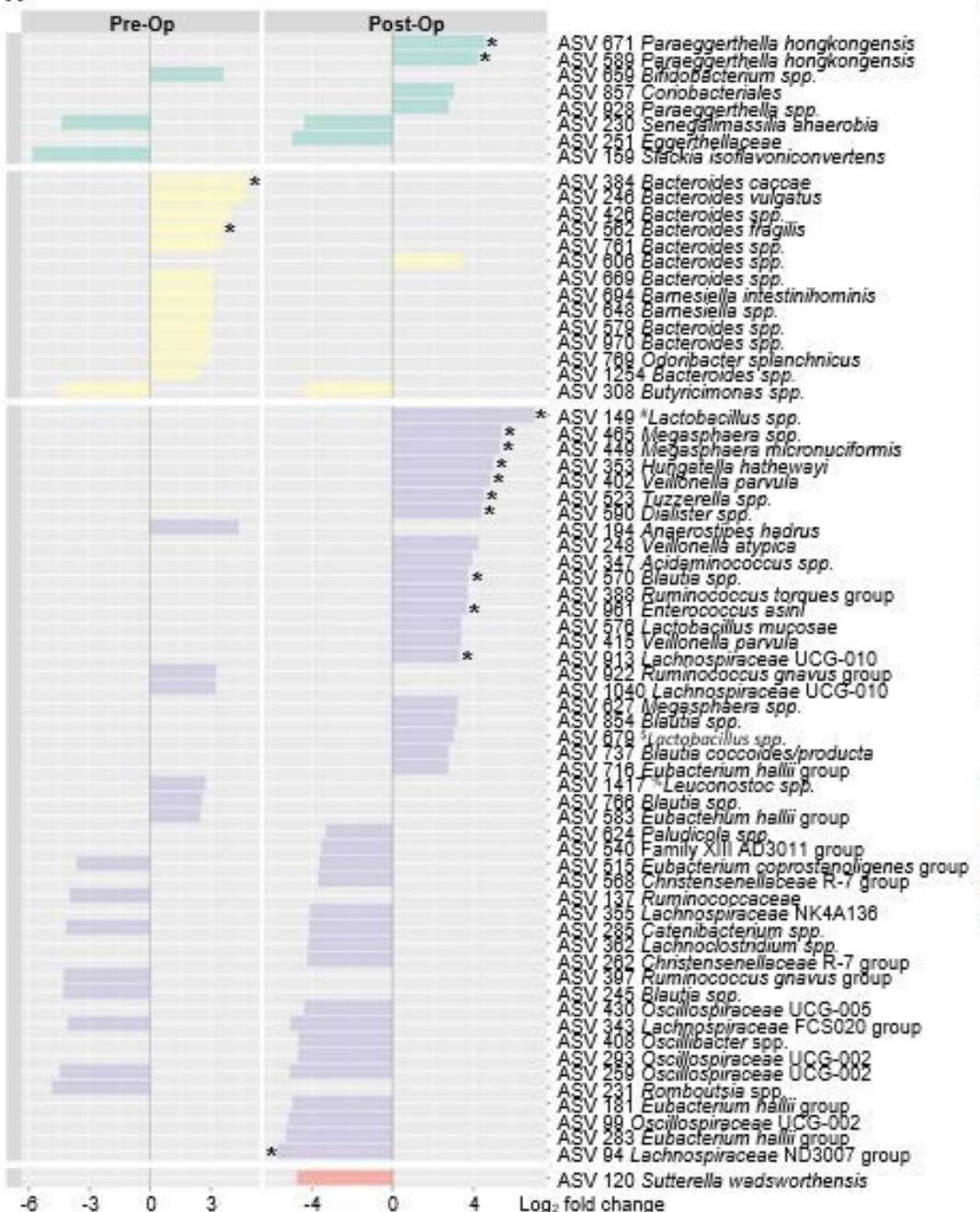

B

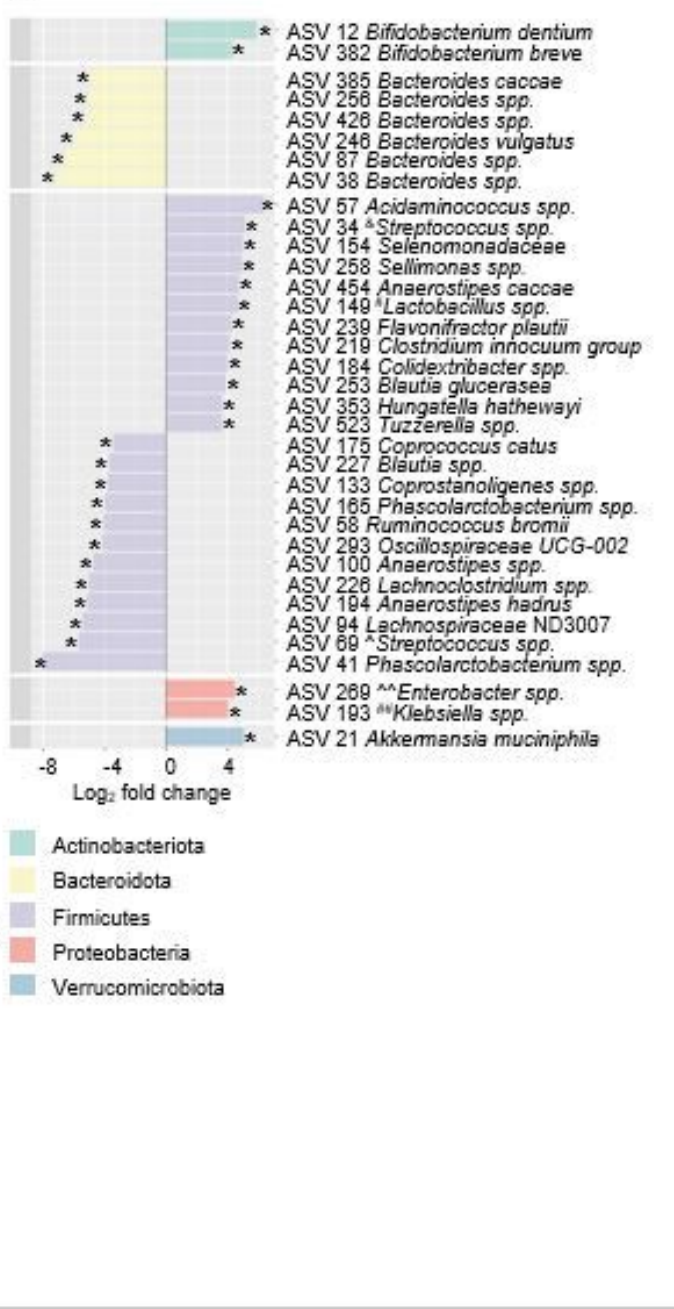

\section{Figure 3}

$<p>A b u n d a n c e$ of specific bacterial ASVs are different in CRC patients compared to NC control and changes after surgery. (A) Bar chart shows bacterial ASVs that are significantly different in CRC patient pre- and post-op compared to

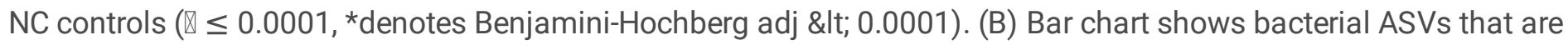
significantly different in CRC post-op compared to pre-op (Paired test, $\mathbb{X} \leq 0.0001$, *denotes Benjamini-Hochberg adj \&lt; 0.0001) $</ p><p>$ Abbreviated ASV annotation as below; $</ p><p>A S V 149 \#<$ em $>$ Lactobacillus acidophilus/casei/crispatus/gallinarum</em>;</p><p>ASV679 $\$<$ em>Lactobacillus</em> $<$ em>crustorum/farciminis/formosensis/heilongjiangensis/mindensis/musae/nantensis $</$ em $>;</ p><p>A S V 1417 \%$ $<$ em>Leuconostoc carnosum/citreum/garlicum/holzapfelii/lactis $</$ em $>;</ p><p>A S V \_34$ \&amp; $<$ em $>$ Streptococcus alactolyticus/equinus/gallolyticus/macedonicus/pasteuri/pasteurianus $</ \mathrm{em}>$; ASV_6 ${ }^{\wedge}{ }^{\wedge}<\mathrm{em}>$ Streptococcus lutetiensis/salivarius/thermophilus $</$ em $>;</ p><p>A S V \_269^{\wedge \wedge}<$ em $>$ Enterobacter aerogenes/asburiae/bugandensis/cancerogenus/cloacae/hormaechei/ludwigii/mori/roggenkampii/soli/tabaci</em>; ASV_193 \#\#<em>Klebsiella pneumoniae/quasivariicola/variicola</em $></ p>$ 
A

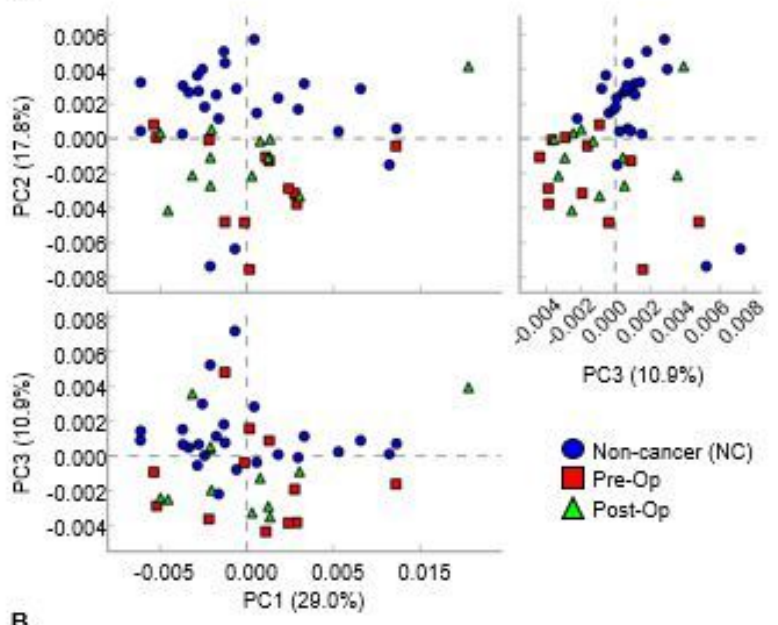

B

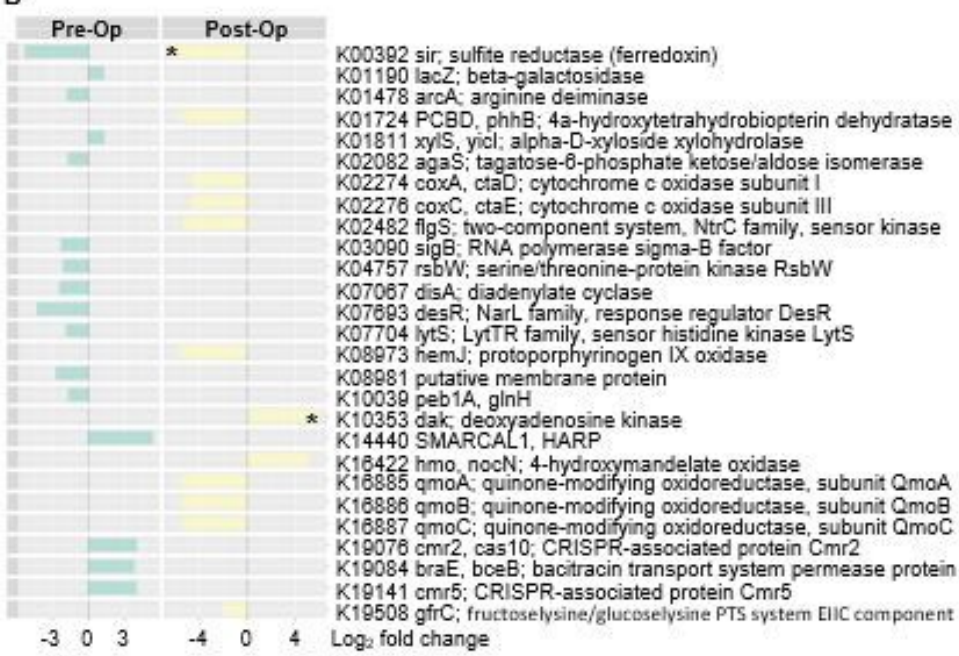

C

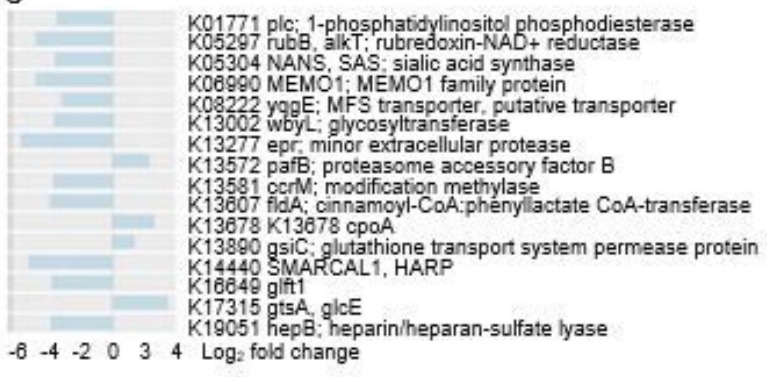

\section{Figure 4}

$<p>$ Changes in functional profile of bacterial composition in NC and CRC patients. (A) Principal coordinate analysis (PCoA) of PICRUSt2-projected functional profiles (level 3, KEGG orthology (KOs)) between NC, CRC patients pre- and post-op (ANOVA Tukey-Kramer post hoc comparison, Benjamini-Hochberg correction, padj\&lt; 0.05). (B) Bar chart shows top differentially represented KOs in CRC patients pre- and post-op compared to NC control ( $\mathbb{Q} \& \mathrm{lt}$; 0.000001 , all comparisons Benjamini-Hochberg adj \&lt; 0.001, * $\mathrm{p}$. adj \& t $\mathrm{t}$ 0.0001). (C) Bar chart shows top differentially represented KOs in CRC post-op compared to pre-op (Paired test, p \&lt; 0.001).</p>

\section{Figure 5}

$<p>$ KEGG gene enrichment analysis. (A, B) Dot plots showing KEGG pathways that were enriched in CRC pre- and postop compared to NC controls respectively. (C) Dot plot shows enriched KEGG pathways in CRC patients pre- and postop. p. adjust and counts denote Benjamini-Hochberg adjusted $p$ values and gene counts respectively. $</ p>\langle p\rangle\langle b r\rangle</ p\rangle$

\section{Supplementary Files}

This is a list of supplementary files associated with this preprint. Click to download.

- SupplementaryData3.xlsx 
- SupplementaryData1.xlsx

- SupplementaryData2.xIsx

- SupplementaryFigure2.pptx

- SupplementaryFigure1.pptx 